\title{
Initial experience with novel Embocure Plus microspheres for transarterial chemoembolization (TACE) of liver metastatic colorectal cancer tumours - a clinical and in vitro study
}

\author{
Piotr Piasecki ${ }^{1 A, B, C, D, E, F}$, Marek Wierzbicki ${ }^{1 B, C, D, E}$, Aleksandra Majewska ${ }^{2 B, C, D, F}$, Claudine Kieda ${ }^{2 D, F}$, Jerzy Narloch ${ }^{1 B, C, D, E}$ \\ 'Department of Interventional Radiology, Military Institute of Medicine, Warsaw, Poland \\ ¿2aboratory of Molecular Oncology and Innovative Therapies, Military Institute of Medicine, Warsaw, Poland
}

\section{Abstract}

\begin{abstract}
Purpose: Transarterial chemoembolization (TACE) is currently recommended for unresectable intrahepatic tumours with no vascular invasion or metastasis to other organs. It is based on drug-eluting microspheres pre-loaded with chemotherapeutics, which are injected selectively into vessels supplying the tumour, to embolize them inducing ischaemia, and elute the drug, to induce tumour response. We present our initial experience with novel irinotecanloaded Embocure Plus microspheres in patients with metastatic colorectal cancer tumours in the liver, and their effect on HCT-116 cell cultures in vitro.
\end{abstract}

Material and methods: Three consecutive male patients (median age 62 [50-76] years) with liver metastatic colorectal cancer tumours were selected. All patients had a pre-procedure contrast-enhanced computed tomography, confirming multiple metastatic liver tumours (mean tumour diameter $=42 \mathrm{~mm}$; range: 14-77 $\mathrm{mm}$ ) and periprocedural dyna-CT scans for rapid treatment results assessment. In vitro: Human colon HCT116 cancer cell line was cultured, irinotecan loaded Embocure Plus microspheres were added. Cultures were assessed after 24 hours and 72 hours of incubation in normoxia or hypoxia.

Results: All embolizations were technically successful, and no complications were observed. Stabilization of the targeted metastatic liver tumours in all patients was noted. In vitro: Significant decrease of the growth of HTC 116 cell lines were observed in controls compared to cells treated with Embocure Plus loaded with irinotecan in normoxia and hypoxia after 48 and 72 hours. We observed a tendency for less inhibited cell proliferation in low-oxygen conditions.

Conclusions: TACE therapy of liver metastatic tumours shows satisfactory results and a low complication rate. Embocure Plus microspheres are safe and technically feasible for superselective chemoembolization of metastatic colorectal cancer liver tumour. Dyna-CT can be used for assessment of treatment results during repeated TACE procedures.

Key words: microspheres, liver tumour, TACE, transarterial chemoembolization, Embocure Plus.

\section{Introduction}

The fast development of technology in interventional radiology, and the improvement of medical devices and intravascular techniques gives wider possibilities for the treatment oncologic patients. A special group of patients requiring effective treatment are those with advanced neoplastic process, especially with liver metastases. Multiple strategies for treating liver metastases are available for its management, including surgical removal, chemotherapeutic drugs, and ablative or chemoembolization procedures $[1,2]$.

Transarterial chemoembolization (TACE) is currently recommended for unresectable intrahepatic tumours with no vascular invasion or metastasis to other organs [1]. It is based on drug-eluting beads (DEBs) - microspheres

\section{Correspondence address:}

Piotr Piasecki, Department of Interventional Radiology, Military Institute of Medicine, 128 Szaserow St., 01-141 Warsaw, Poland, e-mail: ppiasecki@wim.mil.pl

Authors' contribution:

A Study design · B Data collection · C Statistical analysis · D Data interpretation - E Manuscript preparation · F Literature search · G Funds collection 
of a known diameter pre-loaded with chemotherapeutics, which are injected selectively into vessels supplying the tumour, embolizing them and inducing ischaemia, and eluting the drug, to induce tumour response $[3,4]$. Classically, DEBs are designed to be loaded with positively charged chemotherapeutics, which is not the case with novel microspheres. Embocure Plus (EC+) [3,5] can be loaded with Irinotecan and Doxorubicin, due to their sponge-like structure, independently of the chemical properties of the drug. This unique quality gives EC+ microspheres the advantage of being saturated with other currently available chemotherapeutic agents (e.g. Oxaliplatin, Leucovorin), thus offering the possibility to treat many other liver metastatic tumours, nowadays disqualified from TACE. However, novel microspheres require further in vitro and clinical studies. Simultaneously, EC+ spheres absorb contrast medium, which enhances their visibility (as they are deposited in tumours) on dyna-CT examination - a fact that yields a promising option for rapid assessment of early effects of TACE.

In this study, we present our initial experience with trans-arterial chemoembolization using Embocure Plus microspheres in patients with metastatic colorectal cancer tumours in the liver. The aim of the study was to evaluate the effectiveness and safety of embolization, as well as the technical aspects of the procedure. We assessed the drugbinding capacity of the microspheres, their influence on the effectiveness of embolization, and the effect on colon cancer cells in vitro. To our knowledge, there are no published data on Embocure Plus microspheres.

\section{Material and methods}

\section{Clinical data}

Three consecutive patients $(M=3$, median age 62 [50-76] years) with liver metastatic colorectal cancer tumours were selected. All patients $(n=3)$ had a pre-procedure imaging (multiphase computed tomography of the abdomen), confirming multiple metastatic liver tumours (mean tumour diameter $=42 \mathrm{~mm}$; range: $14-77 \mathrm{~mm}$ ). Selective TACE was performed via the radial or common femoral artery. All procedures were performed using Embocure Plus (Balton, Warsaw, Poland) microspheres, which are PVA based, calibrated particles of $90-125 \mathrm{~mm}$ diameter. We used for each procedure $\mathrm{EC}+$ microspheres loaded with $100 \mathrm{mg}$ Irinotecan, and a 100\% dose was superselectively administered.

Tumour vascularization was assessed by diagnostic angiography, and administration of microspheres was performed with a microcatheter - Prograte (Terumo, Tokyo, Japan) - through a direct superselective injection. All embolizations were technically successful, and no complications were observed. In order to assess the effectiveness of the procedure and possible complications, postprocedural dyna-CT scans were performed.

\section{Dyna-CT image collection}

A GE dyna-CT protocol was used for the study after calibration of a C-arm of Innova 4100 (GE) in anteriorposterior and lateral position. The region of interest dynaCT acquisition was focused on the patients' liver. The examination parameters were as follows: rotation angle of C-arm: $20 \%$, rate of images capture: $15 \mathrm{fr} / \mathrm{s}$, total time: $27 \mathrm{~s}$.

Only clearly separated metastatic tumours were measured in dyna-CT liver images during three TACE procedures (initial treatment, last treatment). RECIST criteria were used for treatment result assessment.

\section{Phantom study}

The IEC Body Phantom was used. Microspheres for phantom study were prepared according to the manual. Briefly, the syringe with microspheres was filled with aqua/ iohexol-350 suspension to $20 \mathrm{ml}$, shaken for 5 minutes, and left for a further 60 minutes. Afterwards, the solution was ejected through a sterile filter needle. Before placing in the phantom, spheres were placed in the dedicated vessel and filled up with saline to $40 \mathrm{ml}$.

The biggest sphere ( $37 \mathrm{~mm}$ in diameter, $\mathrm{V}-25.5 \mathrm{ml}$ ) was filled with mixture of Embocure Plus and contrast medium. A second sphere $(28 \mathrm{~mm}$ in diameter, $\mathrm{V}-11.5 \mathrm{ml})$ was filled contrast medium only (50\% mixture of saline and iohexol-350).

The IEC Body Phantom underwent CT examination using a typical abdominal protocol.

\section{Cell line}

Human colon HCT116 cancer cell line was cultured in McCoy's medium (Lonza, Basel, Switzerland) supplemented with $10 \%$ foetal bovine serum (Biovest, Tamba, USA), $100 \mathrm{units} / \mathrm{ml}$ of penicillin, $100 \mu \mathrm{g} / \mathrm{ml}$ of streptomycin, and $25 \mu \mathrm{g} / \mathrm{ml}$ of amphotericin B (Antibiotic-Antimycotic, ThermoFisher Scientific, Waltham, Massachusetts, USA). Cells were passaged at $80 \%$ confluence by detaching with Trypsin (0.25\%) EDTA solution (VWR International, Radnor, Pennsylvania, USA). Cells were mycoplasma free as assayed by PCR Mycoplasma Test (PromoCell, Heidelberg, Germany).

\section{Cell culture}

3400 cells $/ \mathrm{cm}^{2}$ were seeded on culture cell 96-well plates and allowed to adhere to the culture surface. After 24 hours the medium was changed to pre-balanced normoxia and hypoxia, and the cells were placed in normoxic conditions $\left(\mathrm{N}\right.$, i.e. $\left.\sim 19 \% \mathrm{O}_{2}\right)$ in a standard incubator $\left(37^{\circ} \mathrm{C}, 5 \%\right.$ $\mathrm{CO}_{2} / 95 \%$ air $)$ or in hypoxic conditions $\left(\mathrm{H}\right.$, i.e. $\left.1 \% \mathrm{O}_{2}\right)$ in a Xvivo X3 workstation (Biospherix, Parish, New York, USA) $\left(37^{\circ} \mathrm{C}, 5 \% \mathrm{CO}_{2} / 95 \% \mathrm{~N}_{2}\right)$ for another $48 \mathrm{~h}$. Then the medium was changed again, and $10 \%$ volume of 

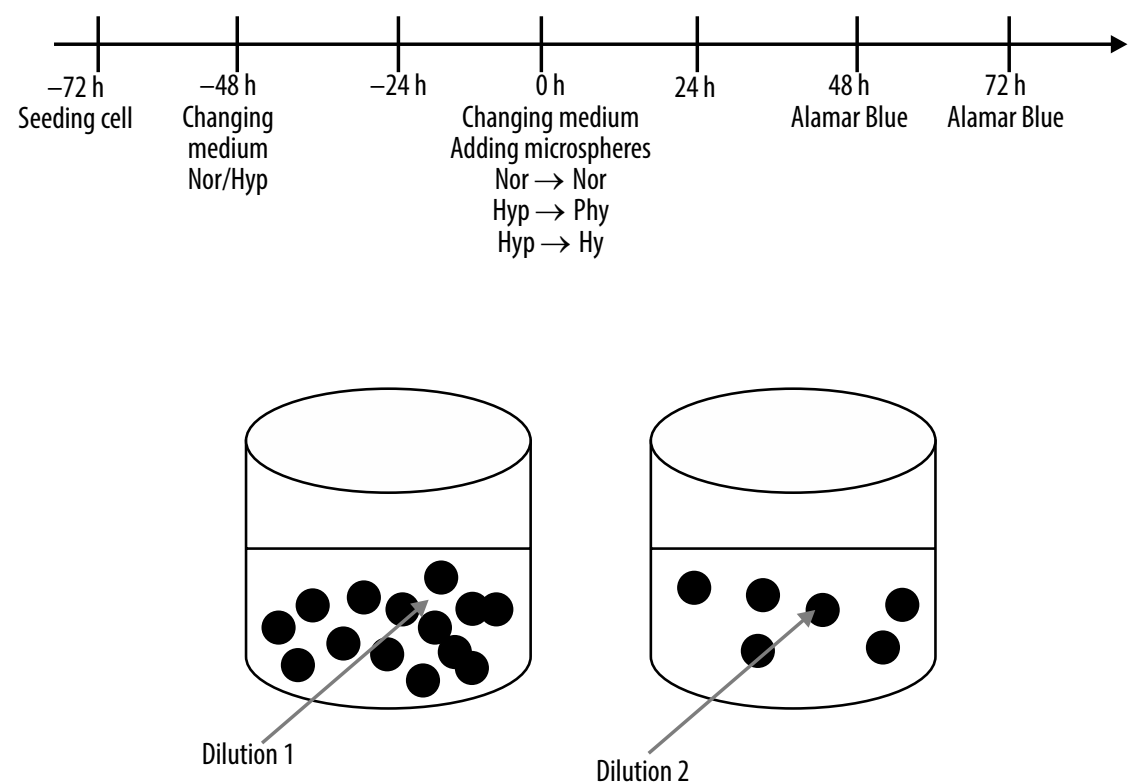

Figure 1. Experiment protocol ( $\mathrm{N}$ - normoxia $19 \% \mathrm{O}_{2^{\prime}} \mathrm{P}$ - physioxia $5 \% \mathrm{O}_{2^{\prime}} \mathrm{H}$ - hypoxia $1 \% \mathrm{O}_{2}$ )

EMBOCURE Plus Microspheres (pre-loaded with $100 \mathrm{mg}$ irinotecan) was added at two different dilutions. Additionally, the supernatant from microspheres with irinotecan was added to the cells to check how quickly the drug is released from the spheres. Cells were cultured for 48 hours or 72 hours under normoxic conditions, with spheres pretreated in normoxia or appropriately pretreated in hypoxia under physioxic or hypoxic conditions [6]. The experiment protocol is represented as a scheme in Figure 1.

\section{Alamar Blue assay}

Alamar Blue reduction assay (G-Bioscience, USA) was performed after 48 hours and 72 hours of culture on cells treated by EMBOCURE Plus microspheres, according to the manufacturer's instructions. Absorbance was measured after 3 hours in a plate spectrophotometer (MultscanGO, ThermoFisher Scientific, Waltham, Massachusetts, USA) at 570 and $600 \mathrm{~nm}$. The percentage of Alamar Blue reduction was calculated compared to cells without microspheres (shown as a percentage of the controls).

\section{Microscopic observations}

Cells were visualized using an inverted microscope Olympus CKX41 with UC30 camera and Olympus Entry Cell Sense 1.8.1. software (Olympus, Tokyo, Japan).

\section{Results}

\section{Tumour response}

We observed stabilization of the size of the targeted metastatic liver tumours in all patients during the TACE treatment in dyna-CT control examinations. One of the patients revealed tumour progression in the control CT examination performed 1 month after 3 courses of TACE (consistent with RECIST 1.1: tumour diameter increased in the left lobe from $35 \mathrm{~mm}$ to $55 \mathrm{~mm}$, and in the right lobe from $76 \mathrm{~mm}$ to $101 \mathrm{~mm}$ ). Two remaining patients are under clinical follow-up, without imaging examination yet (Table 1).

\section{Safety aspects of the procedure}

During follow-up, patients reported complications potentially related to TACE, which were nausea, vomiting, and transient fever. One patient developed an asymptomatic haematoma around the vascular access site. All complications were scored as mild or moderate (Grade 1-2) according to the scale of Common Terminology Criteria for Adverse Events (CTCAE) v 5.0 (Table 1).

\section{In vitro model}

Placing a suspension of loaded microspheres on an HCT 116 cell culture 96-well plate was feasible, safe, and efficient. Embocure plus microspheres without irinotecan had no impact on cell proliferation in culture. A significant decrease of the HTC 116 cell growth was observed when the cells were treated with Embocure Plus loaded with irinotecan in normoxia, hypoxia, and physioxia after 48 hours and 72 hours compared to the controls. The cultured cell proliferation measured by the reduction of $\mathrm{AB}$ after 48 hours and 72 hours was significantly lower in hypoxia, when compared to high oxygen tension.

\section{Dyna-CT}

All targeted tumours were clearly delineated on dyna-CT examination, and their borders reflected tumours seen on contrast-enhanced CT prior to treatment (Figure 5). 
Table 1. Patient characteristics

\begin{tabular}{|c|c|c|c|c|c|c|}
\hline Patient 1 & \multicolumn{2}{|c|}{ Patient 1} & \multicolumn{2}{|c|}{ Patient 2} & \multicolumn{2}{|c|}{ Patient 3} \\
\hline Age (years) & \multicolumn{2}{|c|}{76} & \multicolumn{2}{|c|}{60} & \multicolumn{2}{|c|}{50} \\
\hline Primary tumour histopathology & \multicolumn{2}{|c|}{$\begin{array}{c}\text { Adenocarcinoma tubulare et tubule } \\
\text { - papillare } \mathrm{G} 2 / \mathrm{G} 3\end{array}$} & \multicolumn{2}{|c|}{$\begin{array}{c}\text { Adenocarcinoma tubulare et tubule } \\
\text { - papillare } \mathrm{G} 1 / \mathrm{G} 2\end{array}$} & \multicolumn{2}{|c|}{ Adenocarcinoma tubulare G1/G2 } \\
\hline Primary tumour location & \multicolumn{2}{|c|}{ Ileocecal area } & \multicolumn{2}{|c|}{ Sigmoid colon } & \multicolumn{2}{|c|}{ Sigmoid colon } \\
\hline KRAS status & \multicolumn{2}{|c|}{ Wild type } & \multicolumn{2}{|c|}{ Mutant } & \multicolumn{2}{|c|}{ Mutant } \\
\hline $\begin{array}{l}\text { Number of metastatic tumours } \\
\text { in liver }\end{array}$ & \multicolumn{2}{|c|}{2} & \multicolumn{2}{|c|}{8} & \multicolumn{2}{|c|}{1} \\
\hline $\begin{array}{l}\text { Diameter of the tumours before } \\
\text { the TACE course - CECT (mm) }\end{array}$ & \multicolumn{2}{|c|}{77,37} & \multicolumn{2}{|c|}{$42,76,27,34,35,14,25,23$} & \multicolumn{2}{|c|}{75} \\
\hline $\begin{array}{l}\text { Diameter of the target tumour } \\
\text { after the first TACE treatment - } \\
\text { dyna-CT (mm) }\end{array}$ & \multicolumn{2}{|c|}{78} & \multicolumn{2}{|c|}{43} & \multicolumn{2}{|c|}{75} \\
\hline $\begin{array}{l}\text { Diameter of the target tumour } \\
\text { after TACE course - dyna-CT (mm) }\end{array}$ & \multicolumn{2}{|c|}{79} & \multicolumn{2}{|c|}{$\begin{array}{c}\text { No follow-up examination due to } \\
\text { patient body weight }\end{array}$} & \multicolumn{2}{|c|}{75} \\
\hline $\begin{array}{l}\text { Grade in Common Terminology } \\
\text { Criteria for Adverse Events } \\
\text { (CTCAE v 5.0) after TACE course }\end{array}$ & \multicolumn{2}{|c|}{$\begin{array}{l}\text { Haematoma of the vascular } \\
\text { access site - Grade } 1\end{array}$} & \multicolumn{2}{|c|}{ Fever - Grade 1} & \multicolumn{2}{|c|}{$\begin{array}{l}\text { Nausea - Grade } 1 \\
\text { Vomiting - Grade } 2\end{array}$} \\
\hline \multicolumn{7}{|c|}{ Analysis of patients' laboratory tests } \\
\hline & Before TACE & $\begin{array}{l}\text { After TACE } \\
\text { course }\end{array}$ & Before TACE & $\begin{array}{l}\text { After TACE } \\
\text { course }\end{array}$ & Before TACE & $\begin{array}{l}\text { After TACE } \\
\text { course }\end{array}$ \\
\hline $\mathrm{RBC}\left(\times 10^{12} / \mathrm{I}\right)$ & 4.13 & 3.78 & 4.68 & 4.76 & 4.58 & 4.73 \\
\hline $\mathrm{Hgb}(\mathrm{g} / \mathrm{dl})$ & 13.5 & 12.3 & 15.5 & 15.2 & 13.2 & 12.9 \\
\hline $\mathrm{WBC}\left(\times 10^{9} /\right)$ & 5.6 & 9.14 & 7.32 & 18.09 & 5.0 & 5.27 \\
\hline $\operatorname{PLT}\left(\times 10^{9} / \mathrm{l}\right)$ & 163 & 161 & 189 & 244 & 157 & 129 \\
\hline $\begin{array}{l}\text { Creatinine }(\mathrm{mg} / \mathrm{dl}) / \\
\text { eGFR }\left(\mathrm{ml} / \mathrm{min} / \mathrm{m}^{2}\right)\end{array}$ & $0.9 />90$ & $0.9 />90$ & $0.8 />90$ & $0.71 />90$ & $1.1 />90$ & $1.2 />90$ \\
\hline $\mathrm{K}(\mathrm{mmol} / \mathrm{l})$ & 4.5 & 4.6 & 3.5 & 3.8 & 4.2 & 4.0 \\
\hline $\mathrm{Na}(\mathrm{mmol} / \mathrm{l})$ & 140 & 140 & 141 & 140 & 139 & 141 \\
\hline AST (U/I) & 31 & 51 & 62 & 51 & 32 & 37 \\
\hline ALT (U/l) & 29 & 50 & 72 & 27 & 26 & 25 \\
\hline Total bilirubin (mg/dl) & 0.6 & 0.8 & 0.9 & 0.9 & 0.3 & 0.4 \\
\hline
\end{tabular}

\section{Phantom study}

ROI measurements in a sphere with loaded EC+ showed a mean of $143.4 \mathrm{HU}$ (SD 20.2 HU). In contrast-filled spheres they were 960.7 HU (SD 26.8 HU). For reference, the background density was $-5.6 \mathrm{HU}$ (SD $14.8 \mathrm{HU}$ ).

\section{Discussion}

Efficient intra-arterial treatment of liver tumours comprises stable drug-delivery combined with optimal embolization [1]. This study evaluates initial in vivo and in vitro experience with novel DEBs.

$\mathrm{EC}+$ microspheres introduce novel properties to the spectrum of drug-eluting beads currently available on the market. These beads are composed of spherical, porous, PVA-based particles, which absorb the drug in a sponge- like manner, contrary to ionizable functional groups (carboxylate or sulphonate) [3]. Independence from the chemical properties of loadable drug, theoretically allows higher concentrations, depending on the particle diameter. New drugs can be introduced into TACE treatment, which are unsuitable for loading on conventional DEB.

The loading capabilities are also reflected by absorption of contrast medium. Loaded $\mathrm{EC}+$ spheres are easily visible on angiography when mixed with 50/50 contrast/ saline solution. Cone-beam examination allows confirmation of the microcatheter position before TACE is performed. Sphere deposition in the tumour was distinct in dyna-CT after the procedure [7]. A calibrated phantombased dyna-CT study also proved their clear visibility (Figure 5). Complete infiltration of EC+ spheres in the tumour vascular bed visible on dyna-CT can be a marker of a successful procedure. 

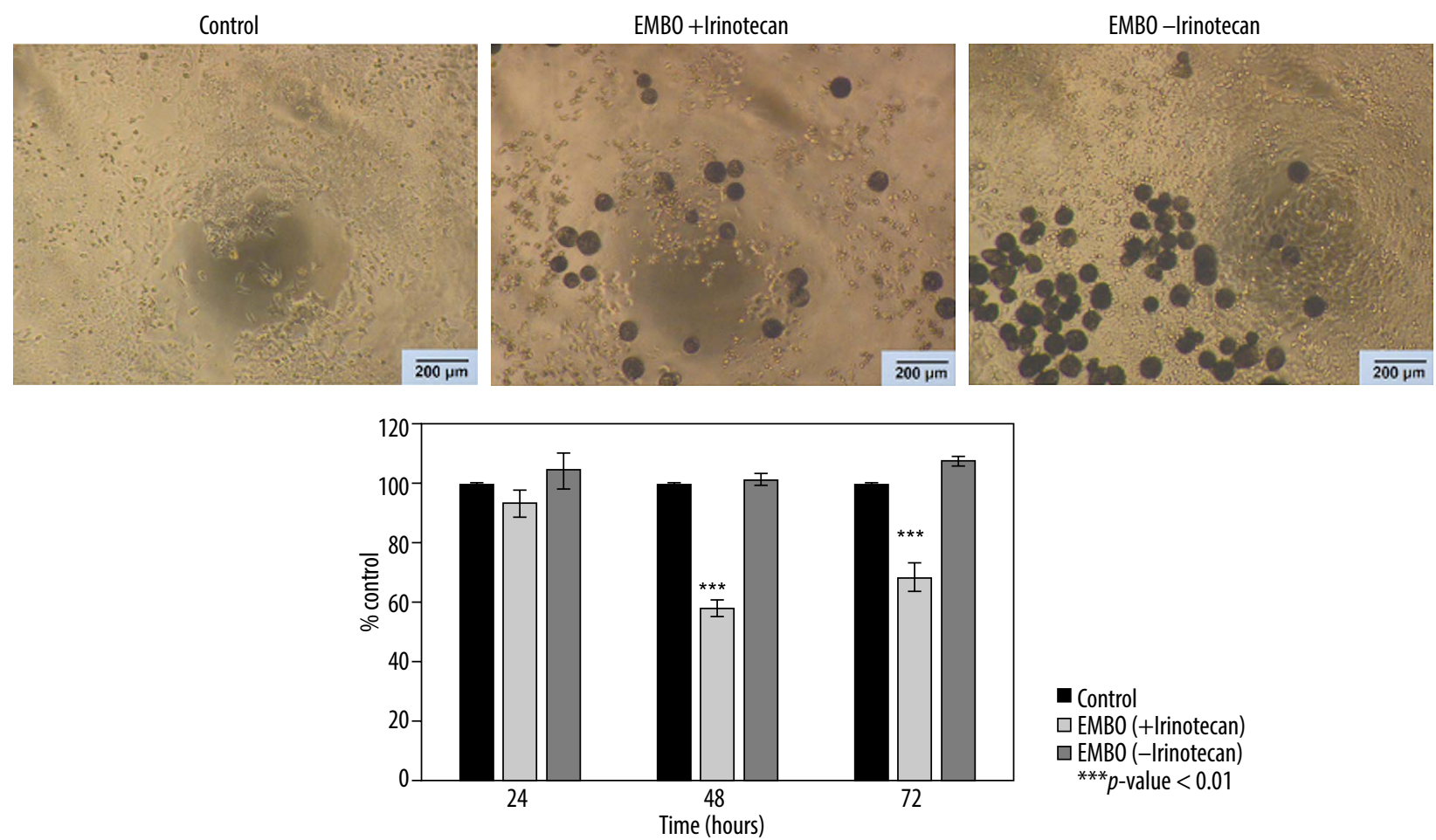

Figure 2. Lack of effect of Embocure Plus microspheres without irinotecan on HCT-116-cultured cell proliferation compared to irinotecan-loaded Embocure Plus microspheres
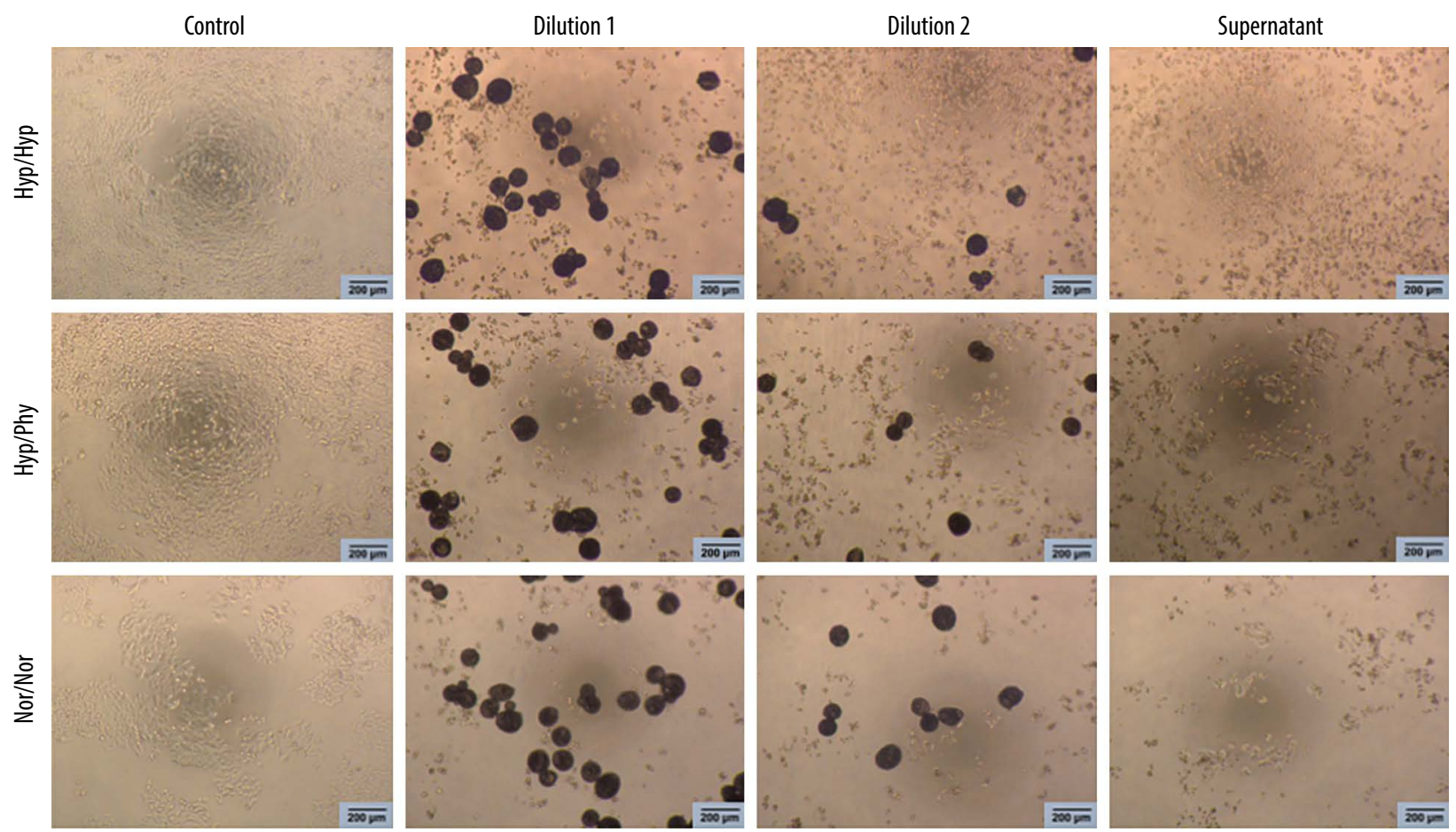

Figure 3. Effect of Embocure Plus microspheres loaded with irinotecan on HCT-116 cultured cells proliferation (reflected by Alamar Blue reduction) for dilution 1, dilution 2 and supernatant after 48 and 72 hours in distinct oxygen tension conditions (normoxia, physioxia and hypoxia)

Recent insight into tumour biology and its inhomogeneous oxygen microenvironment showed that oxygenation conditions inside the tumour are mainly hypoxic $[8,9]$. Hypoxia induces growth of new blood vessels and induces the selection of a hypoxia-resistant tumour [10]. A further decrease in oxygen delivery induced by em- bolization does not lead to tumour cell death, but could induce development of resistance mechanisms against chemotherapeutics $[8,9,11]$.

In each patient, we venture to perform superselective chemoembolization to spare the healthy liver tissue and reduce adverse drug effects, and to minimize devel- 


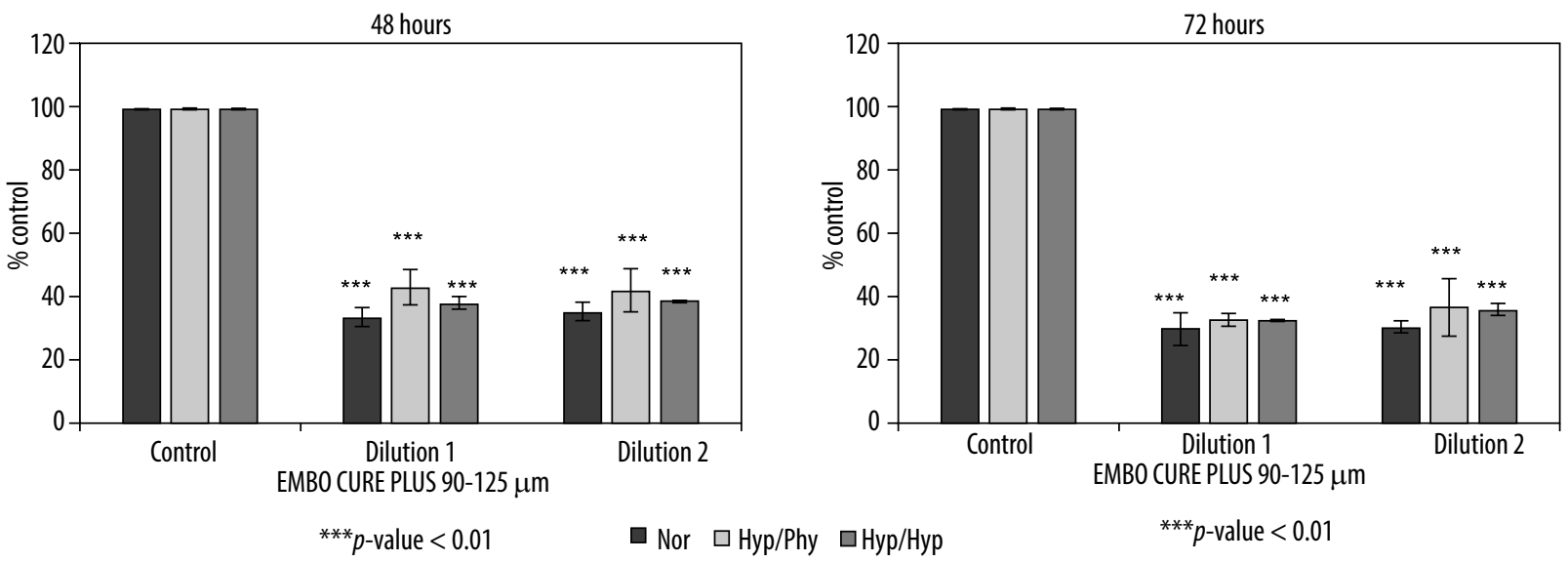

Figure 4. Effect of Embocure Plus microspheres loaded with irinotecan on HCT-116-cultured cell proliferation (reflected by Alamar Blue reduction) for dilution 1 , dilution 2, and supernatant after 48 and 72 hours
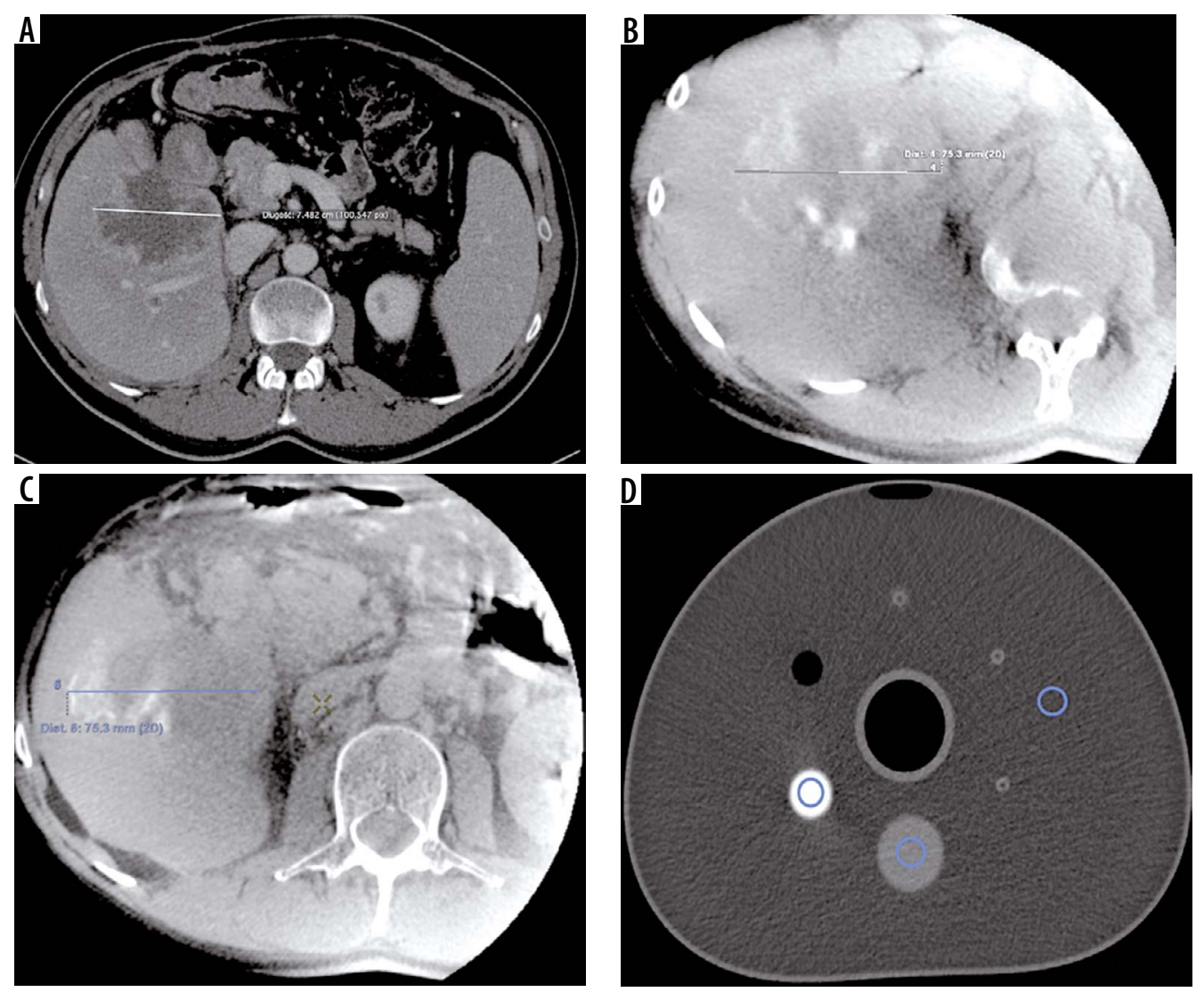

Figure 5. A) Preprocedural CECT multiphase computed tomography of the abdomen revealed a large metastatic tumour in the right lobe of the liver. B) Dyna-CT scan after first TACE procedure shows satisfactory saturation of the tumour with contrast media. C) Dyna-CT scan after last TACE procedure during course with good effects of treatment. D) In vitro phantom used to study microsphere density in embolization mixing (highest density area - contrast media; area of intermediate density - embolization solution with Embocure Plus microspheres; the third ROl is placed in the area of the research environment)

opment of any post-embolization syndrome (Figure 6). Selective embolization is performed until there is considerable blood flow reduction in the afferent vessel, but without any flow arrest. Our observations showed that embolization-generated tumour hypoxia adversely affects the response to treatment. Our in vitro cell-based model of EC+ spheres loaded with irinotecan also supports this phenomenon, in which more colorectal cancer cells were inactivated in more oxygenated conditions (Figures 2-4). During the observation time, the addition of irinotecan- loaded EC+ spheres did not lead to death of all cells, but it was significantly higher compared to controls. Similar observations were made when increasing concentrations of the drug were compared.

In the phantom study, we confirmed that the EC+ microspheres loaded with contrast medium (Omnipaque $350 \mathrm{mg} \mathrm{l} / \mathrm{ml}$ ) resembling TACE conditions were easily distinguished from the phantom body in CT scans. In dyna-CT during TACE, tumour areas saturated with EC+ microspheres were also clearly visible. The use of EC+ 

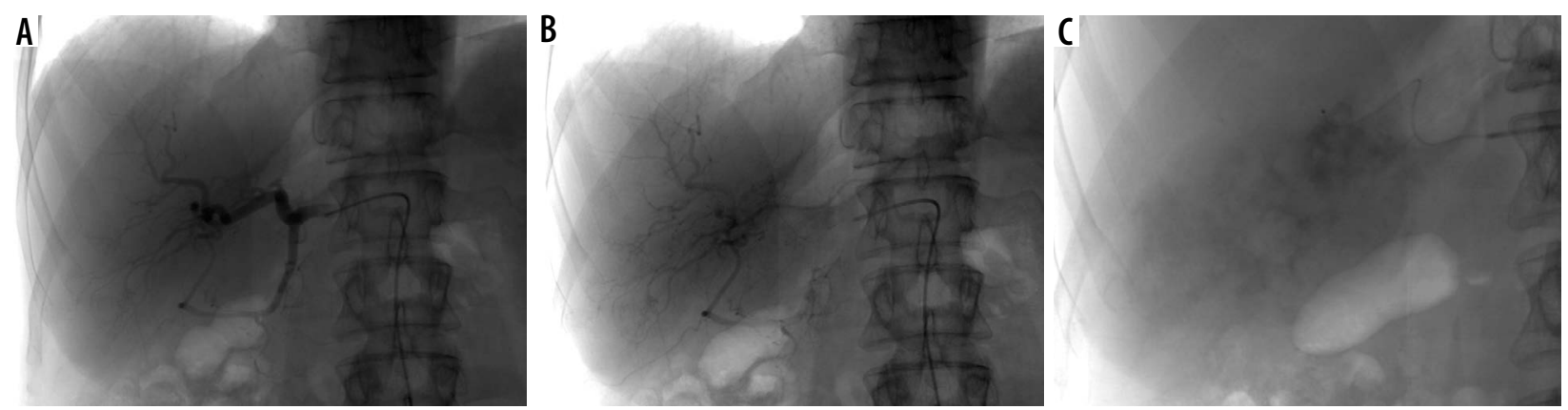

Figure 6. A, B) Intraarterial angiography revealed pathological vascularization of the tumour of the right lobe of the liver from the right hepatic artery. C) Post-superselective chemoembolization angiography shows good infiltration of the tumour with a mixture of microspheres saturated with chemotherapeutic agent (Irinotecan)

microspheres suggested the prospect of clinical success in 3 patients. Our observations showed initial stabilization of tumour growth in repeated dyna-CT exams after each TACE. In one of the patients, on subsequent dyna-CT examinations, less infiltration of microspheres inside the tumour was observed, but the tumour size was stable, which corresponds to tumour vessel damage after each TACE, and to a lower potential microsphere deposition. This phenomenon is known in patients treated with embolics.

Our study has a number of limitations. First, our experience regards a limited number of patients, which foremost affects the impact of clinical observations, and hence conclusions on the overall clinical effects of TACE with $\mathrm{EC}+$ spheres cannot be reliably drawn. Nevertheless, the number of treated tumours is substantial. We based conclusions on the treatment effectiveness on the response of individual tumours, as in RECIST 1.1. A larger cohort may reveal whether the imaging-based result confirm the overall clinical effect on follow-up.

Overall, our observations suggest a place for TACE treatment in metastatic colorectal cancer patients, when combined with other therapies aimed at tumour destruction. Concurrent with most recent ESMO guidelines, we agree that tumour control can be achieved by a multidisciplinary team offering a multimodal, supplemental treatment for each patient [1].

\section{Conclusions}

TACE therapy of liver metastatic tumours shows satisfactory results and low complication rate. Embocure Plus microspheres are safe and technically feasible for superselective chemoembolization of metastatic colorectal cancer liver tumour. Dyna-CT can be used for the assessment of treatment results during repeated TACE procedures. More data are needed to confirm the clinical effectiveness of the preliminary results.

\section{Conflict of interest}

The authors report no conflict of interest.

\section{References}

1. Van Cutsem E, Cervantes A, Adam R, et al. ESMO consensus guidelines for the management of patients with metastatic colorectal cancer. Ann Oncol 2016; 27: 1386-1422.

2. Adam R, de Gramont A, Figueras J, et al. Managing synchronous liver metastases from colorectal cancer: a multidisciplinary international consensus. Cancer Treat Rev 2015; 41: 729-741.

3. Martin RC, Robbins K, Tomalty D, et al. Transarterial chemoembolisation (TACE) using irinotecan-loaded beads for the treatment of unresectable metastases to the liver in patients with colorectal cancer: an interim report. World J Surg Oncol 2009; 7: 80.

4. Iezzi R, Kovacs A, Prenen H, et al. Transarterial chemoembolisation of colorectal liver metastases with irinotecan-loaded beads: what every interventional radiologist should know. Eur J Radiol Open 2020; 7: 100236.

5. Richardson AJ, Laurence JM, Lam VW. Transarterial chemoembolization with irinotecan beads in the treatment of colorectal liver metastases: systematic review. J Vasc Interv Radiol 2013; 24: 1209-1217.
6. McKeown SR. Defining normoxia, physoxia and hypoxia in tumours-implications for treatment response. Br J Radiol 2014; 87: 20130676.

7. Wang C, Wang Q, Chen W, et al. DYNA CT arteriographic evaluation of hepatocellular carcinoma for treatment by trans-catheter arterial chemoembolization. Int J Clin Exp Med 2015; 8: 20548-20555.

8. Graham K, Unger E. Overcoming tumor hypoxia as a barrier to radiotherapy, chemotherapy and immunotherapy in cancer treatment. Int J Nanomedicine 2018; 13: 6049-6058.

9. Carreau A, El Hafny-Rahbi B, Matejuk A, et al. Why is the partial oxygen pressure of human tissues a crucial parameter? Small molecules and hypoxia. J Cell Mol Med 2011; 15: 1239-1253.

10. Welford SM, Giaccia AJ. Hypoxia and senescence: the impact of oxygenation on tumor suppression. Mol Cancer Res 2011; 9: 538-544.

11. Span PN, Bussink J. The role of hypoxia and the immune system in tumor radioresistance. Cancers (Basel) 2019; 11: 1555. 